\title{
sciendo
}

\section{FREEZE DRYING AS A METHOD OF LONG-TERM CONSERVATION OF MAMMALIAN SEMEN - A REVIEW*}

\author{
Iwona Rajska \\ Department of Reproductive Biotechnology and Cryoconservation, \\ National Research Institute of Animal Production, 32-083 Balice n. Kraków, Poland \\ Corresponding author: iwona.rajska@iz.edu.pl
}

\begin{abstract}
With the development of biotechnological methods that allow the manipulation and free exchange of genetic material, the methods for collecting and storing such material need to be improved. To date, freezing in liquid nitrogen has allowed the storage of cells and entire plant and animal tissues for practically unlimited times. However, alternatives are still being sought to eliminate the constant need to maintain samples at a low temperature. Lyophilization or freeze drying is an alternative to standard freezing procedures. The storage of samples (lyophilisates) does not require specialised equipment but only refines the preservation method itself. In the case of cells capable of movement e.g., sperm, they lose the ability to reach the oocyte in vivo and for in vitro fertilization (IVF) because of the lyophilization process. However, freeze-dried sperm may be used for in vitro fertilization by intracytoplasmic sperm injection (ICSI), based on the results obtained in cleavage, embryo development and the production of live born offspring after embryo transfer. Studies on the lyophilization of sperm have been performed on many animal species, both in the laboratory and in livestock. This conservation method is considered to create biobanks for genetically valuable and endangered species with the simultaneous application of ICSI. This review article aimed to present the issues of the freeze-drying process of mammalian semen and help find solutions that will improve this technique of the long-term preservation of biological material.
\end{abstract}

Key words: lyophilization, sperm, ICSI, mammalian

\section{History of freeze-drying}

With the development of biotechnology, more efficient and less labour- and timeconsuming methods of the long-term preservation of biological material have been sought. Cryopreservation is commonly used - i.e., the storage of cells or fragments of plant or animal tissues at liquid nitrogen temperature $\left(-196^{\circ} \mathrm{C}\right)$ (Gajda and Rajska, 2014). This method has many advantages, such as unlimited sample storage time, complete retention of the biological activity of cells and tissues, and the ability

*This review was supported by the Statutory Activity of National Research Institute of Animal Production, project no. 01-19-01-21. 
to store material from genetically valuable organisms (Kasai and Mukaida, 2004; Saragusty and Arav, 2011). However, as with any method, it also has drawbacks - specialised containers and continuous monitoring are necessary to store frozen material in liquid nitrogen so that uncontrolled thawing of samples is avoided. If microbiological purity is not maintained, the pathogenic microorganisms may also be preserved in the frozen material, contributing to subsequent tissue infections. Additionally, the process of freezing and thawing itself, associated with the transformation of water into ice and vice versa, leads to the formation of ice crystals, which can significantly damage cellular organelles, thereby leading to the sample destruction (Holt, 2000). To avoid this undesirable phenomenon, cryopreservation methods were sought in which water passes from liquid to glassy (Saragusty and Arav, 2011). This phenomenon is called vitrification and is an alternative to the standard freezing procedure. The vitrification process often uses a lowered temperature of liquid nitrogen and very small volumes of vitrification fluids to cool biological materials such as embryos, oocytes, somatic cells or whole tissues as quickly as possible (Gajda et al., 2007, 2011, 2015; Hongxia et al., 2018; Dos Santos Morais et al., 2019). Nevertheless, to perform vitrification, appropriate equipment and access to liquid nitrogen and constant monitoring in containers to store vitrified material are necessary.

Lyophilization may be an alternative to the cryopreservation of biological material. This method, although it comprises freezing, is mainly based on the process of freeze drying. The samples (lyophilisates) obtained in this way can be stored under any temperature conditions without needing specialised equipment (containers, ultralow-temperature freezers) and continuous monitoring of their storage temperature.

Benedict in 1905 and Shackell in 1909 performed the first tests of lyophilization of biological material using the vacuum itself and then the vacuum pump. The improvement of this preservation method led to the first bacterial lyophilisates (Hammer, 1911), followed by animal tissues, drugs, blood serum and antibiotics (Flosdorf and Mudd, 1935). In the 1950s, freeze-drying began to be used in the food industry to preserve animal products (e.g., milk, and eggs), vegetable products (e.g., coffee and fruit juices), and other food products (soups, and yeast) (Ramirez and Canizares, 2003).

The breakthrough discovery that initiated the improvement in the lyophilization method in somatic cell preservation was reported by Loi et al. (2008). Blastocysts were obtained because of the introduction of lyophilized granulosa cells, stored at room temperature for 3 years into enucleated sheep oocytes.

The first attempts of semen lyophilization were performed by Polge (1949) on bird spermatozoa. After rehydration, 50\% sperm motility was obtained. Subsequent studies examined the susceptibility of human (Sherman, 1954) and bull semen (Bialy and Smith, 1957) to freeze-drying, but no positive results were obtained. It was not until 1998 that Japanese scientists, Wakayama and Yanagimachi, obtained live offspring in mice using freeze-dried spermatozoa.

In addition to sperm lyophilization, attempts have also been made to lyophilize bovine oocytes. Oocytes were subjected to various cryopreservation procedures (slow freezing, rapid freezing or vitrification) and then were lyophilized. After rehydration, the percentage of live and dead oocytes was evaluated. The highest percent- 
age of live oocytes was obtained because of vitrification and subsequent freeze-drying $(77 \%)$, while the lowest was obtained after using slow freezing and freeze-drying (14\%) (Patrizio et al., 2012).

In studies of sperm lyophilization, the focus was not only on trying to obtain embryos at the blastocyst stage after intracytoplasmic sperm injection and live-born offspring, but also examining the effect of freeze-drying on sperm. Many studies have discussed preserving the ability to fertilize an oocyte despite the loss of sperm motility (Wakayama and Yanagimachi, 1998; Kaneko et al., 2003; Ward et al., 2003). Thus, live offspring were obtained after fertilization with freeze-dried sperm and embryo transfer, as already mentioned, in mice (Wakayama and Yanagimachi, 1998), rats (Hirabayashi et al., 2005), rabbits (Liu et al., 2004), horses (Choi et al., 2011) and hamsters (Muneto and Horiuchi, 2011).

Lyophilization as long-term preservation method for biological material initiated studies of other methods in which the removal of water from animal or plant cells or tissues occurs through assisted evaporation. Examples of such methods are microwave-assisted drying and evaporative drying. To date, using the microwave-assisted drying technique, attempts have been made to preserve tissues such as testicles (Silva et al., 2020) or domestic cat ovaries (Lee et al., 2019). Appropriately prepared tissues are fixed, incubated in a trehalose solution and then dried at an appropriate temperature for a specified time. The study findings showed the acquisition of preserved tissue fragments with a preserved, normal morphology, a low percentage of tissues with damaged DNA and a high percentage of viable cells in the tissue (Lee et al., 2019; Silva et al., 2020).

Attempts have been made to microwave dry feline oocytes in the GV stage (Elliot et al., 2015). The authors investigated the effect of water loss and oocyte storage time on DNA integrity in the GV oocyte stage. As with tissues, oocytes were incubated in the presence of trehalose and then subjected to microwave-assisted drying. DNA fragmentation assessment using the TUNEL method revealed an increase in the percentage of GV cells with damaged DNA in the initial period of oocyte storage; however, subsequently, this percentage remained constant. The obtained results indicated the possibility of preserving the genetic material, without needing to freeze the entire cell, while maintaining a minimum humidity that does not damage cells and allows them to be stored at low temperature or ambient temperature (Elliott et al., 2015).

The second, already mentioned method of preserving biological material, is drying cells or tissues using devices such as laboratory dryers, in which the water contained in the biological material is evaporated. This technique i.e., evaporative drying, has been used in the long-term preservation of cells (e.g., mouse or boar sperm) (Liu et al., 2014; Li et al., 2017). Based on the obtained results, the observed loss of water from boar sperm by evaporation reduced the effectiveness of in vitro fertilization by ICSI. To improve the effectiveness of ICSI using this sperm type, the addition of tauroursodeoxycholic acid (TUDCA), an endogenous bile acid, was used, with a positive effect on embryonic development by reducing the ROS production and the percentage of DNA fragmentation in boar sperm (Li et al., 2017). In turn, Liu et al. (2014) obtained the live offspring of mice by ICSI and sperm preserved by evaporative drying and stored for several months to 2 years at $4^{\circ} \mathrm{C}$ or RT. The percentage of 
blastocysts obtained by ICSI with sperm dried and stored under the abovementioned conditions was slightly higher for sperm stored at $4^{\circ} \mathrm{C}$ than for sperm stored at RT.

Based on the obtained results, evaporative drying of sperm is an alternative to traditional techniques for the long-term preservation of biological material without requiring freezing or freeze-dried sperm (Liu et al., 2014; Li et al., 2017).

\section{Stages of the lyophilization process}

Lyophilization is freeze drying, which involves the initial freezing of the material, followed by eliminating water, which is solvent, by direct ice transition to the gas state bypassing the liquid phase. Freeze-drying is usually performed under reduced pressure, which accelerates the process. The lyophilization process is complex and multistage. First, the material is frozen under atmospheric pressure. Next, vacuum sublimation of the ice from freezing is performed. The final stage is drying the material by heating, which allows removal of the remaining water that has not turned into ice (Narbutt et al., 2017).

As already mentioned, the first stage of freeze-drying is freezing or less often vitrification. The water contained in the material forms ice crystals, increasing the concentration and viscosity of the liquid phase. The result is a high-viscosity, intercrystalline solution. Further lowering of the temperature stops the formation of ice crystals between which a glass-like structure is formed (Narbutt et al., 2017).

The second stage of lyophilization is the freeze-drying phase. It involves removing previously frozen water from the material by its direct transition into steam, or sublimation. This step requires energy in the form of heat, which in a strictly controlled manner, should lead to the removal of steam as soon as possible to prevent damage to the dried material. The elimination of water vapour occurs during mass diffusion exchange with the pressure difference between the material and frozen surface of the condenser. Drying is the most time-consuming stage of freeze-drying, thus it is important to optimize the parameters of this phase. Under suboptimal conditions, the entire drying process can be significantly extended, negatively affecting the quality of the lyophilisate obtained. The temperature of the target product, the rate at which it is achieved by the dried material and maintaining it at a constant level throughout the entire freeze-drying stage, play crucial roles here. The entire drying process is performed under reduced pressure in the freeze dryer chamber, accelerating the transition of frozen water into steam (Narbutt et al., 2017).

The third and last stage of lyophilization is desorption i.e., the second freezedrying or drying. This stage allows the remaining moisture content to be reduced to a minimum level (usually less than 1\%). To achieve this effect, the temperature of the material being dried is slowly increased, with materials being preferably dried briefly and at a higher temperature (e.g., 3-6 hours, at $30^{\circ} \mathrm{C}$ ) (Narbutt et al., 2017).

Several types of freeze dryers are available: centrifugal, shelf and collector. In large laboratories and biotechnology or pharmaceutical companies, large freeze dryers with an ice condenser capacity of up to $1500 \mathrm{~kg}$ are used. Centrifugal freeze dryers evenly remove water from the material, while shelf freeze dryers do not have moving parts, and the material in appropriate packaging (test tubes, glass vessels) is placed on the shelves. These types of freeze dryers are most often used in smaller laboratories (Figure 2). 


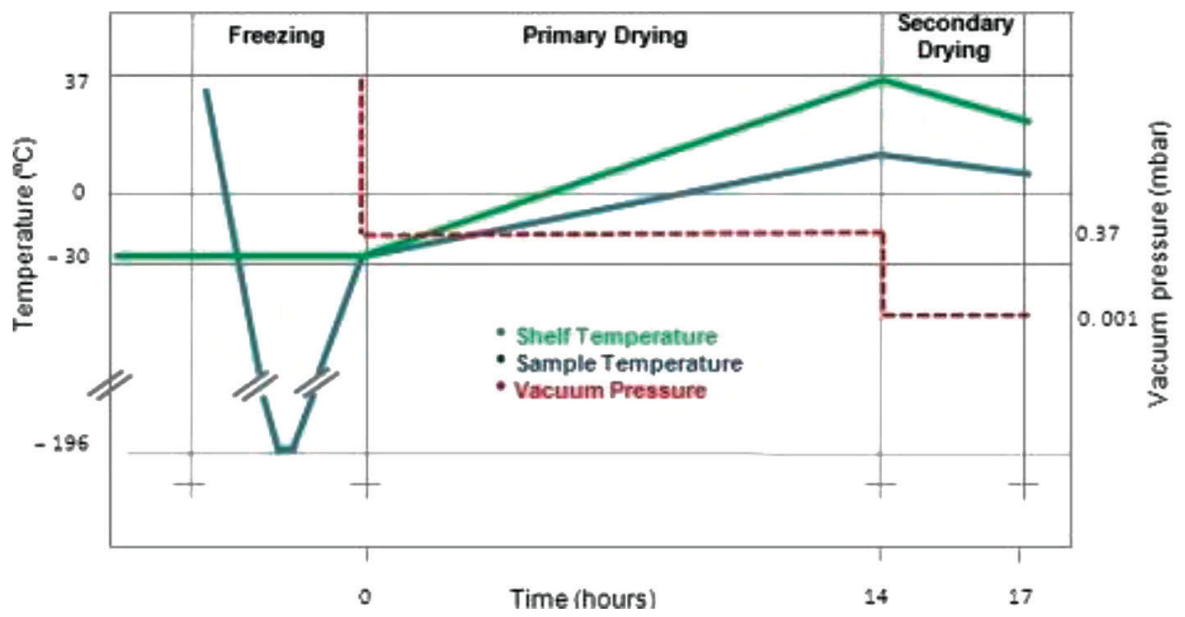

Figure 1. Schematic of an example protocol for lyophilizing sperm in a lyophilizer: time, temperature and pressure (Olaciregui et al., 2014)

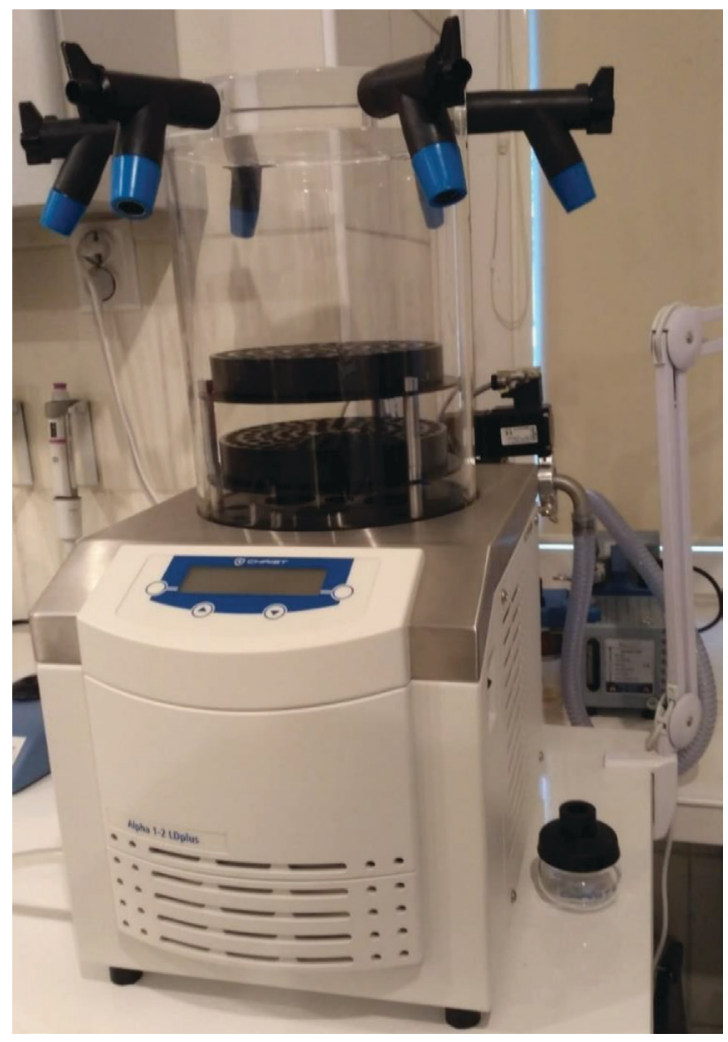

Figure 2. Freeze-dryer Alpha model 1-2 LD plus (prod. Martin Christ Gefriertrocknungsanlagen GmbH - Germany) used in our laboratory for freeze-drying boar semen 


\section{Freeze-drying of animal semen}

The preparation of the semen for the lyophilization process comprises the following stages: collection and pretreatment of the semen (dilution and macro- and microscopic evaluation of the semen), preparation of the lyophilization medium, dilution and freezing of the semen in the lyophilization medium, and lyophilization in the apparatus (appropriate time, temperature and pressure parameters). After the lyophilization process, the samples are stored and rehydrated, and then semen quality is assessed after lyophilization and used for in vitro fertilization by ICSI (Rajska, 2015).

The current state of knowledge regarding research on the freeze-drying of sperm for laboratory, farm, pet and wild animals is presented based on the most important modifications of the lyophilization method and achievement of research teams.
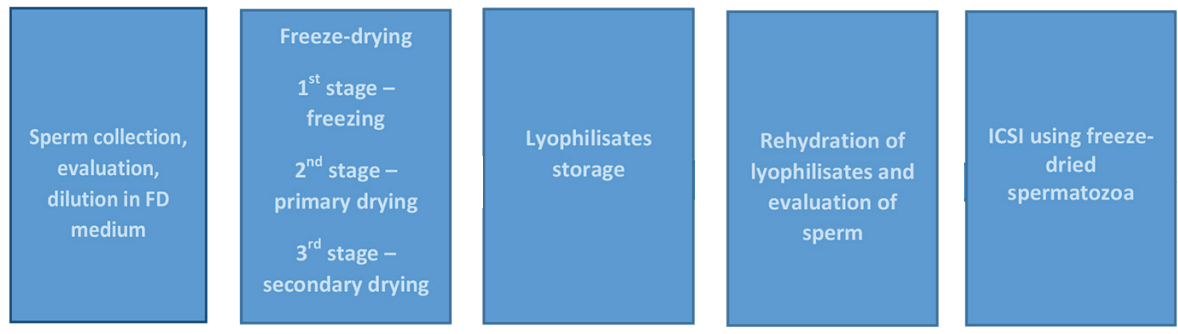

Figure 3. Schematic diagram of the semen lyophilization process

\section{Laboratory animals}

Easy breeding, a short reproductive cycle and high susceptibility to biotechnology indicate that mice have become a graceful and most commonly used species for sperm lyophilization research. To date, mice have been used in most experimental trials and have provided the highest results for in vitro fertilization by ICSI method and the number of liveborn animals after embryo transfer. In mice, epididymal semen is mainly used for lyophilization (Wakayama and Yanagimachi, 1998; Kusakabe et al., 2001; Kusakabe and Tateno, 2017; Kamada et al., 2018; Ito et al., 2019; Kusakabe, 2019); less often spermatozoa are obtained directly from postmortem testicles (Kaneko et al., 2003). After initial microscopic evaluation, semen is diluted in a lyophilization medium most often TRIS-HCl+EGTA (ethylenetetraacetic acid) (Kusakabe et al., 2001; Kusakabe and Tateno, 2017) with various supplements (e.g., diothiothreitol (DTT), diamide (Kaneko et al., 2003), trehalose, sodium ions $\left(\mathrm{Na}^{+}\right)$and/or potassium $\left(\mathrm{K}^{+}\right)$(Kusakabe and Tateno, 2017). Less often, Chatot-Ziomek-Bavister medium (CZB) is used without the addition of chelating compounds such as EDTA (ethylenediaminetetraacetic acid) (Wakayama and Yanagimachi, 
1998; Kusakabe et al., 2001; Wakayama et al., 2017), CZB+HEPES (Kusakabe et al., 2001), and Dulbecco's modified Eagle's medium (DMEM) with the addition of foetal bovine serum (FBS) (Wakayama and Yanagimachi, 1998), TRIS-HCl+EDTA (Kaneko, 2015) and human tubal fluid (HTF) medium (Kamada et al., 2018). Next, the semen samples are subjected to one-stage (Kusakabe et al., 2001; Wakayama et al., 2017, 2019; Kamada et al., 2018; Ito et al., 2019; Kusakabe, 2019), rarely twostage, freeze-drying (Bhowmick et al., 2003; Kawase et al., 2007 a, b), using a constant pressure from $0.022 \mathrm{mBar}$ to $120 \mathrm{mBar}$ (Kusakabe and Tateno, 2011, 2017) or a variable pressure in the range from 0.04-1.03 to $0.001 \mathrm{mBar}$ (Kawase et al., $2007 \mathrm{a}$, b) during a cycle and temperature from $-50^{\circ} \mathrm{C}$ to $0^{\circ} \mathrm{C}$ (Wakayama and Yanagimachi, 1998; Kawase et al., 2009). The freeze-drying time is from 3 to 19 hours (Wakayama and Yanagimachi, 1998; Kusakabe et al., 2001; Kamada et al., 2018; Ito et al., 2019; Kusakabe, 2019; Wakayama et al., 2019). The lyophilisates obtained are stored at low temperatures e.g., $-80^{\circ} \mathrm{C}$ (Kawase et al., 2005), $-30^{\circ} \mathrm{C}$ (Wakayama et al., 2017), $4^{\circ} \mathrm{C}$ (Wakayama and Yanagimachi, 1998; Kaneko, 2015; Kusakabe, 2019), room temperature (RT) (Kamada et al., 2018; Ito et al., 2019; Kusakabe, 2019) or an incubator temperature, e.g. $+30^{\circ} \mathrm{C}$ (Kawase et al., 2007 a) or $+50^{\circ} \mathrm{C}$ (Kusakabe and Tateno, 2011). Only glass ampoules are used to store the samples of lyophilized semen (Kusakabe et al., 2001; Wakayama et al., 2017, 2019; Kusakabe, 2019); the samples may be additionally protected with aluminium foil (Wakayama and Yanagimachi, 1998) or packed in a paper box and plastic bag (Kamada et al., 2018). After reconstruction of lyophilized mouse sperm, the degree of DNA damage in sperm is most often assessed using the comet assay (Kawase et al., 2005, 2007 a, 2009; Kusakabe and Tateno, 2011; Wakayama et al., 2017, 2019; Kamada et al., 2018), and gammaH2AX (Kamada et al., 2018; Ito et al., 2019) and acridine orange staining (Kawase et al., 2009). Additionally, sperm morphology and damage caused during the freezedrying process are assessed by scanning electronic microscopy (SEM) (Wakayama et al., 2019); molecular analysis of microsatellites is also performed (Wakayama et al., 2017).

In subsequent experiments, the harmful effects of freeze-drying on the nuclear chromatin of spermatozoa are observed. To minimize these adverse phenomena, chemical compounds are sought to stabilize sperm DNA.

For this purpose, chelates such as EGTA or EDTA have been used to evaluate the degree of sperm chromatin integrity in mice (Kaneko and Nakagata, 2005; Kaneko et al., 2009; Nakagawa and Kaneko, 2019). These chemical compounds inhibit the activity of endogenous nucleases that leak out of the spermatozoa because of damage to their cytoplasmic membranes and destroy the DNA structure causing the degradation of sperm chromosomes in mice (Kaneko and Nakagata, 2005; Kaneko et al., 2009; Nakagawa and Kaneko, 2019). Similar observations were made by Bhowmick et al. (2003) comparing the effect of EGTA supplementation on the stability of mouse sperm nuclear chromatin and the high $\mathrm{pH}$ of this compound solution. The obtained results suggested that high $\mathrm{pH}$ and the presence of EGTA caused a decrease in the activity of endogenous nucleases (Bhowmick et al., 2003). By contrast, the high concentration of potassium ions $\left(\mathrm{K}^{+}\right)$in the lyophilization medium promoted the stabilisation of mouse sperm DNA, preventing damage to chromosomes in sperm 
stored at high temperatures (Kusakabe, 2019). Additionally, the use of incorrect pressure values during the first stage of freeze drying affected the integrity of sperm proteins and caused a decrease in their biological functionality (Kawase et al., $2007 \mathrm{a}, \mathrm{b})$.

In mice, the efficiency of sperm lyophilization is mainly assessed based on the percentage of fertilized oocytes, obtained blastocysts, and liveborn pups. Additionally, the effectiveness of the lyophilization process is analysed based on sperm evaluation after rehydration. In mice, the percentage of live sperm (with intact chromatin) ranges from 6\% to 100\% (Kawase et al., 2009; Kawase and Suzuki, 2011). The percentage of embryo cleavage in mice ranges from approximately $40 \%$ to approximately $95 \%$, while that of obtained blastocysts ranges from $50 \%$ to approximately 90\% (Wakayama and Yanagimachi, 1998; Kaneko and Nakagata, 2005; Kaneko, 2015; Kusakabe, 2019). The number of litters produced after ICSI using freeze-dried spermatozoa ranges from 9 to 57, with an average of 4 pups/recipient (Kamada et al., 2018; Ito et al., 2019; Wakayama et al., 2019).

In rats, for lyophilization, spermatozoa are mainly obtained postmortem, from the epididymis (Hirabayashi et al., 2007; Kaneko et al., 2007; Hochi et al., 2008; Kaneko et al., 2009; Kaneko and Serikawa, 2012; Nakagawa and Kaneko, 2019). After macro- and microscopic evaluation and dilution to the selected concentration of spermatozoa, the lyophilization medium was added: TRIS-HCl+EDTA (Kaneko et al., 2007; Hochi et al., 2008) or interchangeably +EGTA (Kaneko et al., 2007, 2009; Kaneko and Serikawa, 2012; Nakagawa and Kaneko, 2019). Regarding rat semen, most authors use single-stage lyophilization (Hirabayashi et al., 2007; Kaneko et al., 2007, 2009; Kaneko and Serikawa, 2012; Nakagawa and Kaneko, 2019), while two-stage was applied only by Hochi et al. (2008). The duration of the entire cycle is usually 4 hours (Kaneko et al., 2007, 2009; Kaneko and Serikawa, 2012; Nakagawa and Kaneko, 2019), although some use longer freeze-drying times (Hirabayashi et al., 2007; Hochi et al., 2008) and constant pressure values ranging from 0.02 to 0.095 mBar (Kaneko et al., 2009; Kaneko and Serikawa, 2012; Nakagawa and Kaneko, 2019), while variable pressure values ranging from 0.037 to $0.001 \mathrm{mBar}$ are used (Kaneko et al., 2007; Hochi et al., 2008) and temperatures ranging from $-30^{\circ} \mathrm{C}$ to $+30^{\circ} \mathrm{C}$ (Hochi et al., 2008). Rat sperm lyophilisates are usually stored at $4^{\circ} \mathrm{C}(\mathrm{Hi}-$ rabayashi et al., 2007; Kaneko et al., 2007; Hochi et al., 2008; Kaneko et al., 2009; Kaneko and Serikawa, 2012; Nakagawa and Kaneko, 2019), less frequently RT or at liquid nitrogen temperature $\left(-196^{\circ} \mathrm{C}\right)$ (Hochi et al., 2008), at different times: from several days to one year (Hirabayashi et al., 2007; Hochi et al., 2008; Kaneko et al., 2009), in plastic cryotubes (Hirabayashi et al., 2007) or glass ampoules (Kaneko and Serikawa, 2012; Nakagawa and Kaneko, 2019). After rehydration, the semen is subjected to a quality assessment including the degree of chromatin dispersion - (using the comet assay with propidium iodide staining (Kaneko and Serikawa, 2012) or SYBR-14 (Kaneko et al., 2009), and the integrity of the sperm cell membrane is assessed using FITC-PNA staining (Hirabayashi et al., 2007).

Similar to that in mice, chelates such as EGTA and EDTA were also used to stabilize the nuclear chromatin of the spermatozoa in rats. The high $\mathrm{pH}$ of the EDTA solution had a positive effect on nuclear chromatin and inhibited endogenous nucleases 
in rat sperm, affecting sperm DNA integrity (Kaneko and Serikawa, 2012; Nakagawa and Kaneko, 2019).

The effectiveness of freeze-drying rat sperm was assessed based on various sperm viability tests, including chromosome sperm analysis. In the study by Hochi et al. (2008) it chromatin damage occurred in approximately $35 \%$ to $100 \%$ of the lyophilized sperm analysed. By contrast, Kaneko (2009) and Kaneko and Serikawa (2012) observed a much higher percentage of spermatozoa with damaged chromatin after freeze-drying and long-term storage (one year) than after shorter storage (6 months).

In rats, embryonic cleavage ranges from $30 \%$ to approximately $70 \%$, the percentage of blastocysts remains approximately $35 \%$ on average (Hirabayashi et al., 2007; Kaneko and Serikawa, 2012; Nakagawa and Kaneko, 2019) and the number of liveborn pups is between 3 and 13 (Hochi et al., 2008; Kaneko et al., 2009; Kaneko and Serikawa, 2012).

Ejaculated semen is mainly used for freeze-drying in rabbits (Liu et al., 2004; Domingo et al., 2018; Mercati et al., 2019). After initial evaluation and dilution using a commercial diluent, the semen was divided into portions and the lyophilization medium was added. TRIS-HCl+EDTA is most commonly used (Domingo et al., 2018; Mercati et al., 2019) without or with the addition of RA (Domingo et al., 2018; Mercati et al., 2019) or melatonin (Mercati et al., 2019). TRIS-HCl was also used in combination with EGTA and RA (Liu et al., 2004; Domingo et al., 2018). The prepared rabbit semen samples were subjected to one-stage (Liu et al., 2004) or two-stage (Domingo et al., 2018; Mercati et al., 2019) lyophilization using a constant pressure of $0.023-0.04 \mathrm{mBar}$ (Liu et al., 2004) or variable pressure during the cycle and from 0.053 to $0.018 \mathrm{mBar}$ (Domingo et al., 2018; Mercati et al., 2019) and a temperature range from $-68^{\circ} \mathrm{C}$ to $+20^{\circ} \mathrm{C}$ (Domingo et al., 2018; Mercati et al., 2019). The duration of one-stage lyophilization was 4 hours (Liu et al., 2004). The lyophilisates obtained were stored in glass ampoules (Liu et al., 2004; Domingo et al., 2018; Mercati et al., 2019) at $4^{\circ} \mathrm{C}$ (Liu et al., 2004; Domingo et al., 2018) or RT (Domingo et al., 2018) for 8 months (Domingo et al., 2018) up to two years, although Liu et al. (2004) stored semen samples after lyophilization for an additional 12-18 months. After reconstruction, lyophilized spermatozoa were subjected to traditional morphological assessment by light microscopy (Mercati et al., 2019) and SEM (Liu et al., 2004; Mercati et al., 2019). Additionally, the degree of chromatin dispersion was also assessed using the comet assay (Domingo et al., 2018), the integrity of the sperm cell membranes was assessed by propidium iodide and SYBR-14 staining (Liu et al., 2004), and the degree of DNA methylation (Mercati et al., 2019) and morphology, and the number of sperm chromosomes were assessed (Liu et al., 2004).

The lyophilization process in rabbit semen, similar to that in mice and rats, caused the loss of sperm motility (Liu et al., 2004). By contrast, changes in the nuclear chromatin were observed in approximately $8 \%$ of freeze-dried spermatozoa (Liu et al., 2004). Domingo et al. (2018) observed a higher percentage of rabbit sperm with intact chromatin in the group of freeze-dried spermatozoa in the presence of EDTA $(3.7 \%-4.7 \%)$ than in the EGTA group $(9.5 \%-12.5 \%)$.

Regarding rabbits, few ICSI attempts have been performed using freeze-dried sperm. The efficiency of embryonic cleavage ranges from $30 \%$ to $70 \%$, while the percentage of blastocysts is approximately $24 \%$ (Liu et al., 2004). 


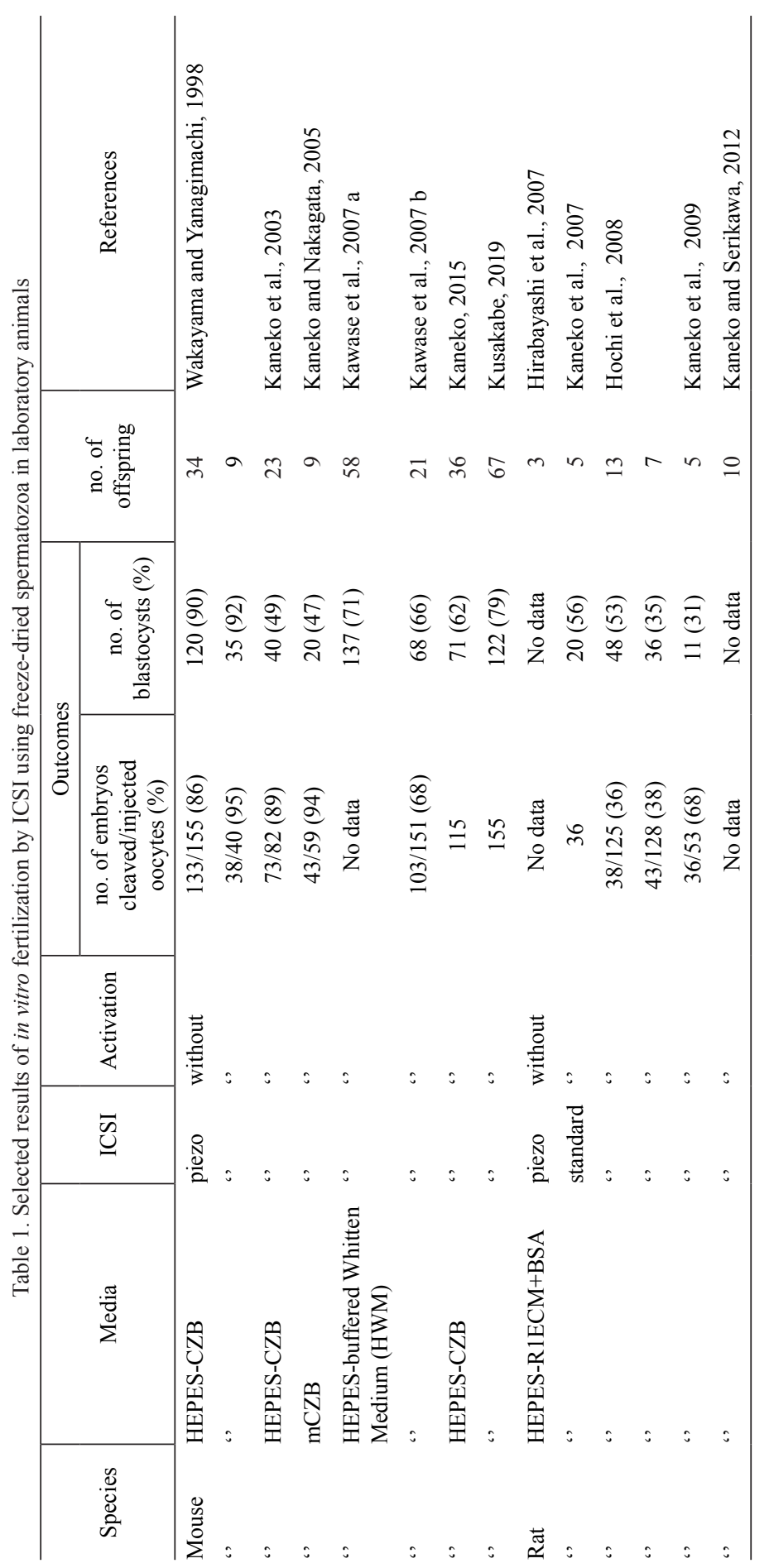


The lower in vitro fertilization efficiency of ICSI in rabbits, compared with that in mice and rats, may be due to the effect of the presence or damage of sperm-borne oocyte activating factor (SOAF) during lyophilization on further oocyte development after fertilization (Liu et al., 2004). The presence of this factor affects the transformation in the cytoplasm of the oocyte, in its absence or with significant damage, activation of the oocyte after injection may not be performed properly. Therefore, to improve the percentage of correctly fertilized oocytes, additional chemical and electrical activation of oocytes were used. Additionally, in rabbit semen, the presence of a compromised sperm cytoplasmic membrane after the lyophilization process may cause chemical changes in the cell membranes or sperm cytoplasm. This phenomenon causes greater difficulties in the biochemical transformation of membranes than in the case of fresh sperm Therefore, the lyophilization process that causes partial or complete damage to sperm cytoplasmic membranes may improve the in vitro fertilization efficiency of ICSI in this species (Liu et al., 2004). To date, because of in vitro fertilization by the ICSI method and after ET, one live young was obtained (Liu et al., 2004) (Table 1).

\section{Farm animals}

Ram semen intended for lyophilization is collected in an artificial vagina (Olaciregui et al., 2017 a; Anzalone et al., 2018; Arav et al., 2018), or spermatozoa are obtained postmortem from the epididymis (Palazzese et al., 2018). Next, the semen is diluted in a commercial diluent and aliquoted to achieve the desired concentration of spermatozoa in a lyophilization medium, e.g., based on a solution of TRIS-HCl + EGTA or TRIS-HCl + EGTA supplemented with RA (Olaciregui et al., 2017 a). Additionally, other authors have used "xeroprotective medium" (Palazzese et al., 2018) or a medium based on TRIS with the addition of trehalose, sorbitol and egg yolk at various concentrations (Arav et al., 2018). The prepared semen samples are intended for one-stage (Arav et al., 2018; Palazzese et al., 2018) or two-stage lyophilization (Olaciregui et al., 2017 a). The ram sperm are lyophilized in the temperature range from $-84^{\circ} \mathrm{C}$ to $+20^{\circ} \mathrm{C}$ (Palazzese et al., 2018; Arav et al., 2018; Olaciregui et al., $2017 \mathrm{a}$ ), while maintaining a constant pressure of $0.11 \mathrm{mBar}$ (Arav et al., 2018) or $0.006 \mathrm{mBar}$ (Palazzese et al., 2018) or variable pressure from 0.053 to $0.018 \mathrm{mBar}$ (Olaciregui et al., 2017 a) for a period of one hour (Arav et al., 2018) to 24 hours (Palazzese et al., 2018). The lyophilisates obtained are stored in glass ampoules (Olaciregui et al., 2017 a; Anzalone et al., 2018; Palazzese et al., 2018) or on slides (Arav et al., 2018), at $4^{\circ} \mathrm{C}$ (Olaciregui et al., 2017 a) or at RT (Olaciregui et al., 2017 a; Anzalone et al., 2018; Palazzese et al., 2018) for one year (Olaciregui et al., 2017 a) or intended directly for fertilization (Anzalone et al., 2018; Palazzese et al., 2018). After rehydration, semen quality assessment was performed and the following parameters were tested: chromatin damage (standard comet or two-tail comet assay) (Olaciregui et al., 2017 a; Palazzese et al., 2018), sperm viability using eosin-nigrosin staining (Palazzese et al., 2018), degree of acrosome integrity (Palazzese et al., 2018), degree of violation of the sperm cytoplasmic membrane - using propidium iodide staining (Anzalone et al., 2018), sperm morphology (Palazzese et al., 2018) and sperm motility using computerassisted sperm microscopic evaluation of the CASA system (Palazzese et al., 2018). 
Unlike other species, in the studies of Arav et al. (2018) on the freeze-drying of ram semen using a modern lyophilization device with very fast sublimation at high subzero temperature, it was possible to achieve sperm motility at the level of even approx. $47 \%$ after sample rehydration. The authors of the study claimed that the combination of appropriate pressure, temperature and sample volume, as well as the optimal composition of the lyophilization medium containing sugars limiting damage resulting from osmotic stress, has allowed such results to be obtained. Nevertheless, the analysis of low-temperature scanning electron microscopy showed much damage within the cytoplasmic membrane of the sperm using the abovementioned lyophilization process (Arav et al., 2018).

One of the tests to assess the integrity of sperm chromatin in freeze-dried ram sperm was the comet assay. Damage to chromatin in freeze-dried semen compared with that in nonfreeze-dried semen ranged from $2.8 \%$ to $95.9 \%$, with most of the damage being DNA single-strand breaks (SSBs) (Olaciregui et al., 2017 a; Palazzasse et al., 2018).

Moreover, as with other species, an RA additive was used, which favourably influenced sperm DNA and helped maintain the integrity of nuclear chromatin (Olaciregui et al., 2017 a). In addition to chelating compounds and antioxidants, additional oocyte activation after injection was used to improve the in vitro fertilization efficiency by ICSI with freeze-dried sperm. This process increased the proportion of cleaved oocytes and obtained blastocysts (Anzalone et al., 2018).

In sheep, the efficiency of embryonic cleavage was approximately $11 \%$ to $37 \%$, while $0 \%$ to approximately $25 \%$ developed to the blastocyst stage. Additionally, in this species, EGTA was added to the lyophilization medium, which inactivated DNAse and protected the sperm DNA from the harmful effects of the lyophilization process and changes during storage of the lyophilisates (Olaciregui et al., 2017 a).

Although sperm freezing in cattle is a common conservation method in this species, alternative methods are sought that do not require specialized equipment and the use of liquid nitrogen. Therefore, similar to the case of pigs, the lyophilization method is being improved in cattle as an alternative to the long-term preservation of male gametes. In cattle, ejaculated semen is usually intended for lyophilization (Martins et al., 2007 a, b), and undergoes initial macro- and microscopic assessment, and then is diluted in a commercial diluent and portioned. The prepared bull semen samples are intended for lyophilization in a medium based on TRIS-HCl solution with selected supplements: EDTA (Martins et al., 2007 a) or EGTA (Abdalla et al., 2009; Hara et al., 2011, 2014). Other media are also used, e.g. DMEM (Keskintepe et al., 2002) or TCM-199 enriched with Hank's salts and foetal bovine serum (FBS) (Martins et al., 2007 a, b). Semen is lyophilized in one (Keskintepe et al., 2002) or two stages (Abdalla et al., 2009) at a constant pressure in the range from 0.19 to $0.35 \mathrm{mBar}$ (Keskintepe et al., 2002; Martins et al., $2007 \mathrm{a}, \mathrm{b}$ ) or a variable pressure of 1.98-0.12 mBar (Hara et al., 2014) or 0.37-0.001 mBar (Abdalla et al., 2009; Hara et al., 2011) and at a temperature of $-47^{\circ} \mathrm{C}$ to $+25^{\circ} \mathrm{C}$ (Keskintepe et al., 2002; Hara et al., 2014) from 6 to 18 hours (Keskintepe et al., 2002; Martins et al., 2007 a, b; Hara et al., 2014). The bull semen lyophilisates obtained are stored in glass ampoules additionally secured with aluminium foil (Hara et al., 2014), at liquid nitrogen tem- 
perature (Abdalla et al., 2009), at $-20^{\circ} \mathrm{C}$ (Hara et al., 2014) or at $4^{\circ} \mathrm{C}$ (Keskintepe et al., 2002; Martins et al., 2007 a, b; Abdalla et al., 2009; Hara et al., 2011) for several days and months to one year (Keskintepe et al., 2002; Martins et al., 2007 a, b; Abdalla et al., 2009; Hara et al., 2011, 2014). After rehydration in lyophilized bull spermatozoa, the following parameters are assessed: the degree of chromatin damage by the comet assay (Hara et al., 2011, 2014), integrity of sperm cell membranes by acridine orange staining (Martins et al., 2007 a, b), sperm morphology using SEM (Martins et al., 2007 b), level of apoptosis in sperm by the TUNEL method (Martins et al., $2007 \mathrm{a}, \mathrm{b})$.

Martins et al. (2007 a) showed that the addition of EGTA protects sperm structures such as the nucleus, mitochondria and acrosome, positively affecting their functionality. Additionally, trehalose in bull lyophilization medium improved the ability to decondensate sperm nuclear chromatin and transform it into a functional male pronucleus, thus increasing the ICSI fertilization efficiency. The addition of both EGTA and trehalose reduced the percentage of sperm with damaged DNA (from $1 \%-2 \%$ to $2 \%-5 \%$, respectively) compared with the percentage of sperm lyophilized in medium without the abovementioned substance $(4 \%-14 \%)$.

In addition to the protective effects on sperm DNA, chelating compounds also support the integrity of the entire sperm cell. The effect of this action is an increased proportion of morphologically normal spermatozoa with no loss of acrosome or tail, which is common after lyophilization (Martins et al., 2007 b). However, freeze-dried sperm in the presence of trehalose or EGTA had stable chromatin $(100 \%$ and 100\%, respectively) and largely intact acrosomes (approx. 90\%) (Martins et al., 2007 b).

Hara et al. (2011) demonstrated that the lyophilization protocol used did not cause significant sperm DNA damage $(21.7 \%)$ compared with the nonfreeze-dried protocol $(23.3 \%)$.

Embryonic cleavage in cattle averaged approximately $45 \%$, while the percentage of obtained blastocysts was very diverse and ranged from a few to approximately $30 \%$. Similar to that in pigs, the in vitro fertilization efficiency of ICSI in cattle is influenced by the presence of an affected sperm cytoplasmic membrane. This effect, which can be caused by immobilisation of the sperm by damage to the switch, positively influences and improves the percentage of fertilized oocytes and further embryonic development (Keskintepe et al., 2002). Additional activation, e.g., by incubating oocytes after injection with ethanol or ionomycin, also positively influences and improves the effectiveness of fertilization by the ICSI method (Keskintepe et al., 2002).

The low ICSI fertilization efficiency in cattle can be caused, similar to that in rabbits (Liu et al., 2004) and pigs (Kwon et al., 2004), by damage to SOAF, which is necessary for further correct activation of the oocyte after injection (Hara et al., 2014), as well as damage and disturbance in the functioning of centrosomes in bull spermatozoa after freeze-drying, leading to a decreased capacity for full embryonic development after fertilization using the ICSI method (Hara et al., 2011).

For many years, research teams worldwide have been conducting experiments to optimise the effective method of the long-term preservation of boar semen. For this 
species, sperm are more sensitive to freezing and thawing factors than those of other farm and laboratory animal species. The cause may be the specific composition of fatty acids and high concentration of polyunsaturated fatty acids in the spermatozoa. The consequence of this phenomenon is increased susceptibility to osmotic stress and damage to the cytoplasmic membrane of the sperm and cellular organelles causing apoptotic and necrotic changes in male gametes (Holt, 2000). The reduced survival of boar spermatozoa after the freezing-thawing process results not only in reduced reproduction rates and frequent reinsemination of sows, but also in a reduced percentage of cleaved embryos and number of blastocysts obtained because of in vitro fertilization. Therefore, more efficient and less complicated methods of boar sperm preservation are constantly being sought. An example of an alternative method to freezing in liquid nitrogen is freeze-drying. To date, embryos and expanding blastocysts have been obtained because of in vitro fertilization by ICSI of freeze-dried spermatozoa. However, this method of sperm preservation is still being improved by searching for suitable additives for freeze-drying media or optimal parameters of the freeze-drying process itself. In pigs, the ejaculate sperm fraction is mainly intended for lyophilization (Kwon et al., 2004; Nakai et al., 2007; Men et al., 2013; Garcia et al., 2014; Rajska, 2015; Olaciregui et al., 2017 b; Gajda et al., 2019). After initial microscopic evaluation, the semen was diluted with a commercial diluent and divided into portions. For lyophilization, media based on TRIS-HCl solution enriched with chelating compounds: e.g., EDTA (Nakai et al., 2007; Garcia et al., 2014; Olaciregui et al., 2017 b) or EGTA (Nakai et al., 2007; Men et al., 2013; Olaciregui et al., 2017 b), and saccharides (e.g. lactose, trehalose, sucrose in various concentrations (Garcia et al., 2014), rosmarinic acid (Olaciregui et al., 2017 b) or sorbitol (Nakai et al., 2007) are usually used. Less commonly used media are based on Tyrode Lactate-HEPES (TL-HEPES) with the addition of polyvinyl alcohol (PVA) (Kwon et al., 2004; Gajda et al., 2019) or without the addition of chelates, (e.g. TRIS-HCl + sorbitol (Nakai et al., 2007). The prepared boar semen samples are subjected to one-stage (Garcia et al., 2014; Gajda et al., 2019) or two-stage lyophilization (Garcia et al., 2014; Men et al., 2013, 2016; Olaciregui et al., 2017 b) at a constant pressure of $0.13 \mathrm{mBar}$ (Men et al., 2013, 2016), $0.37 \mathrm{mBar}$ (Gajda et al., 2019) or $0.039 \mathrm{mBar}$ (Kwon et al., 2004) or a variable pressure in the range $0.01 \mathrm{mBar}-0.015 \mathrm{mBar}$ (Garcia et al., 2014) or $0.053-0.018 \mathrm{mBar}$ (Olaciregui et al., $2017 \mathrm{~b}$ ) and a temperature from $68^{\circ} \mathrm{C}$ to $+30^{\circ} \mathrm{C}$ (Nakai et al., 2007; Garcia et al., 2014; Men et al., 2013, 2016; Olaciregui et al., 2017 b). The freeze-drying time of sperm ranges from 6 to 30 hours (Kwon et al., 2004; Nakai et al., 2007; Garcia et al., 2014; Men et al., 2013, 2016; Olaciregui et al., 2017 b). The lyophilisates obtained are stored in glass ampoules (Nakai et al., 2007; Men et al., 2013: Garcia et al., 2014; Olaciregui et al., 2017 b) or cryoprobes (Kwon et al., 2004; Garcia et al., 2014), at $4^{\circ} \mathrm{C}$ (Kwon et al., 2004; Nakai et al., 2007; Men et al., 2013; Olaciregui et al., 2017 b; Gajda et al., 2019) or in the range from $-80^{\circ} \mathrm{C}$, $-20^{\circ} \mathrm{C}, 4^{\circ} \mathrm{C}$ to $25^{\circ} \mathrm{C}$ (Kwon et al., 2004; Garcia et al., 2014) for one month (Garcia et al., 2014; Gajda et al., 2019), three months (Gajda et al., 2019), half a year (Kwon et al., 2004), one year (Olaciregui et al., 2017 b) or until ICSI (Men et al., 2013). After reconstruction in freeze-dried boar semen, the degree of sperm chromatin damage was assessed by the comet assay (Men et al., 2013; Olaciregui et al., 2017 b) or stain- 
ing with acridine orange (Garcia et al., 2014; Gajda et al., 2019), as well as apoptotic changes in the sperm using the TUNEL method (Nakai et al., 2007).

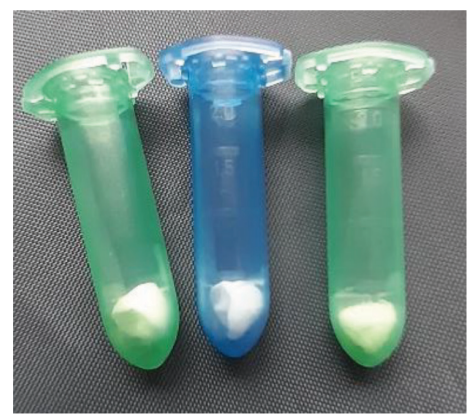

Figure 4. Boar semen lyophilisates produced in our laboratory

The addition of RA to the boar sperm lyophilization medium prevents DNA damage during freeze-drying (Olaciregui et al., 2017 b). Rosmarinic acid (RA) is a powerful antioxidant that effectively neutralises free radicals that cause various types of DNA strand damage. However, the presence of RA did not favourably increase the percentage of cleaved embryos and blastocysts obtained because of ICSI. The authors explained this phenomenon as the lack of influence of a certain level of sperm chromatin damage on the ICSI results using freeze-dried sperm and the ability of oocytes to repair certain sperm DNA damage at later stages of the fertilization process.

Similar observations were made by Men et al. (2016) who studied the effect of trehalose supplementation on the expression level of genes responsible for repairing DNA damage in oocytes. The presence of trehalose had a positive effect, causing a decrease in the percentage of spermatozoa showing DNA fragmentation after the lyophilization process; however, it did not improve the effectiveness of ICSI. Additionally, the ability to repair DNA strand damage depends on the type of damage and cytoplasmic quality of oocytes. More complicated disorders in the structure of sperm chromatin cause irreversible changes and are the cause of chromosomal aberrations, resulting in serious disorders in embryonic development (Men et al., 2016).

As already mentioned, the effectiveness of freeze-dried boar spermatozoa is influenced by the addition of chelating substances such as EGTA or EDTA. Nakai et al. (2007) reported that the presence of EGTA or EDTA in boar sperm lyophilization medium effectively reduced the percentage of spermatozoa, with DNA fragmentation appearing during rehydration and incubation, and better results were obtained using EGTA. By contrast, Olaciregui et al. (2017 b) and Men et al. (2016) showed that the addition of EDTA causes a greater decrease in the percentage of spermatozoa with damaged chromatin after freezing boar sperm.

Investigating the effect of chelates and RA addition on the integrity of the nuclear chromatin of boar sperm, Olaciregui et al. (2017 b) showed the highest percentage 
of chromatin-damaged spermatozoa in the EGTA group (5.1\%) and the lowest in the EGTA group with RA addition $(0.7 \%)$. Similar results were obtained by Nakai et al. (2007), who examined the degree of damage to sperm chromatin in freeze-dried sperm, rehydrated and incubated in the fertilization medium. The highest percentage of spermatozoa with damaged chromatin (approximately 3\%) was observed in lyophilized semen in the presence of fertilization medium (Pig-FM) compared to freeze-dried semen in lyophilization media. In contrast, a significant increase in DFI was observed in lyophilized sperm incubated for 180 minutes in Pig-FM medium (12.2\%) compared to other experimental groups (0.7\%-4.1\%) (Nakai et al., 2007).

By contrast, in Garcia et al. (2014) experiments a higher percentage of spermatozoa showing intact chromatin (95.7\%) was observed in lyophilisates stored in glass ampoules regardless of the storage temperature and type of lyophilization medium used compared with lyophilisates stored in plastic cryotubes $(85.3 \%)$.

By analysing the degree of sperm nuclear chromatin defragmentation, Men et al. (2013) using the comet assay, showed that as the concentration of trehalose in the lyophilization medium increases, the surface of the chromatin dispersion (large halo) increases.

Damage to sperm chromatin arising during the lyophilization process can be eliminated by adding the abovementioned substances. However, a prolonged lyophilization process may negatively affect sperm morphology and cause structural damage, leading to a disturbance of oocyte activation after injection (Kwon et al., 2004).

Our experiments (Gajda, unpublished data) showed that with the increased storage time of lyophilisates, the percentage of sperm with fragmented chromatin increased. Analysing the semen from two boars, freeze-dried in TRIS medium, we observed that more than $50 \%$ of spermatozoa had chromatin damage after 90 days of sample storage at $20^{\circ} \mathrm{C}$. In turn, no significant differences were found in the percentage of spermatozoa with fragmented chromatin in the semen stored at $4^{\circ} \mathrm{C}$ depending on the storage time and the lyophilization medium used (TL-HEPES-PVA, TRIS and glucose-yolk) (Gajda et al., 2019).

Regarding livestock, the efficiency of ICSI is much lower than that for laboratory animals. In the case of pigs, the percentage of embryonic cleavage ranged from approximately $26 \%$ to approximately $80 \%$, and blastocysts from approximately $9 \%$ to approximately $60 \%$. Kwon et al. (2004) suggest that the low percentage of blastocysts obtained by in vitro fertilization by ICSI in pigs may be due to SOAF damage or inactivation during the lyophilization process. Additionally, the same authors point to the low efficiency of ICSI as the cause of damage to sperm chromatin, which can impair the development of a fertilized oocyte.

In our laboratory, the in vitro fertilization efficiency of ICSI using freeze-dried spermatozoa was approximately $26 \%$, while approximately $35 \%$ of embryos reached the blastocyst stage (Rajska, unpublished data). A higher number of blastocysts (30) was obtained because of ICSI using freeze-dried boar semen stored for 30 days than that (14) after fertilization with 90 day stored semen (Rajska, unpublished data). The probable reason for the lower number of blastocysts in the group of oocytes fertilized with sperm stored for 90 days is damage to the sperm nuclear chromatin caused by the storage time of lyophilisates. As already mentioned, both the freeze-drying process and the storage method of the lyophilized sperm samples can affect the efficiency of ICSI. 


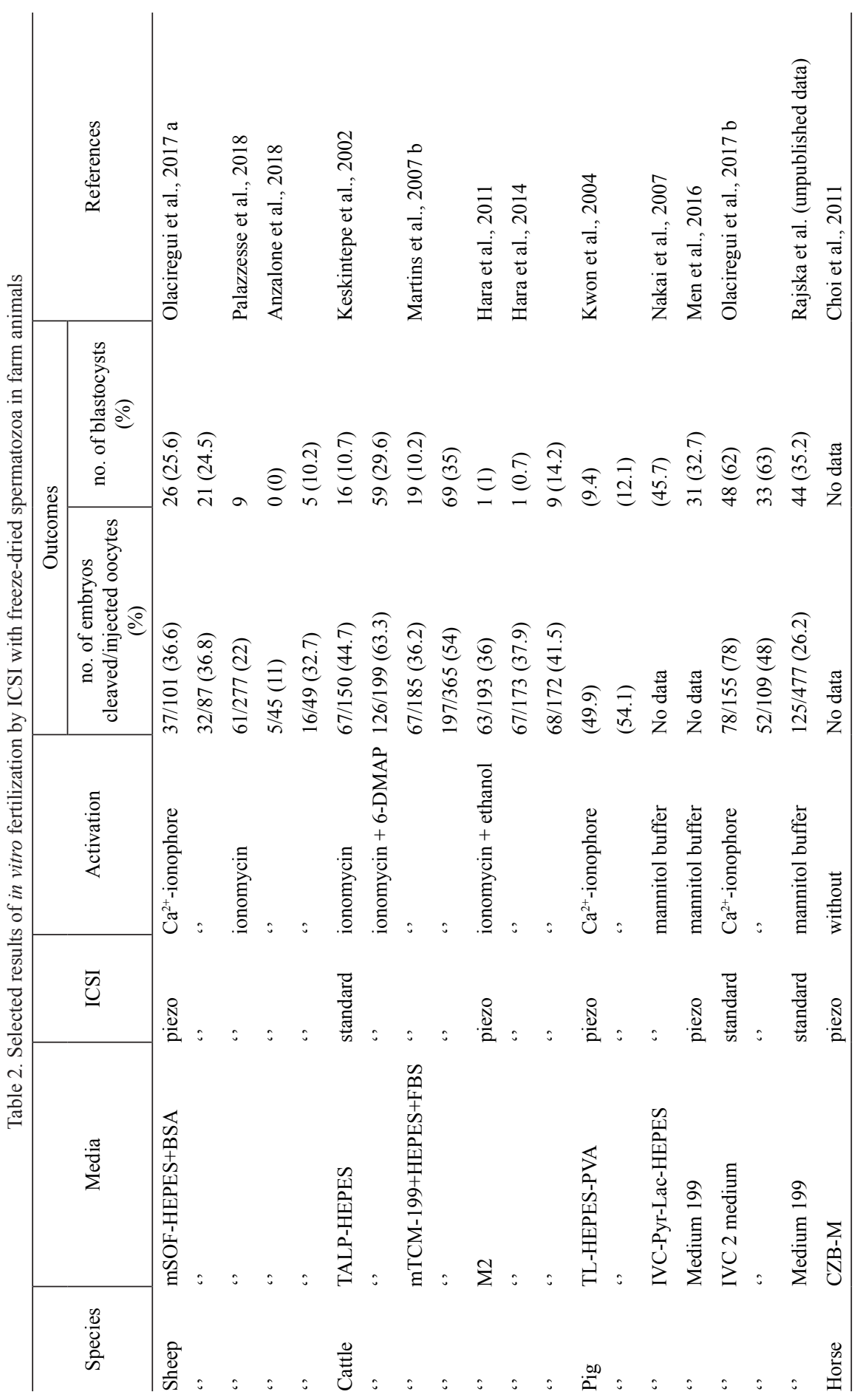


However, the lyophilization process, as has already been mentioned several times, causes damage to the cytoplasmic membranes of spermatozoa, accelerating the initiation of the transformation of the sperm cell nucleus into functional male pronuclei (Rajska, 2015).

To date, because of ICSI and ET, Nakai et al. (2007) two pregnant sows who had abortions on the 29th and 39th days of pregnancy (Table 2).

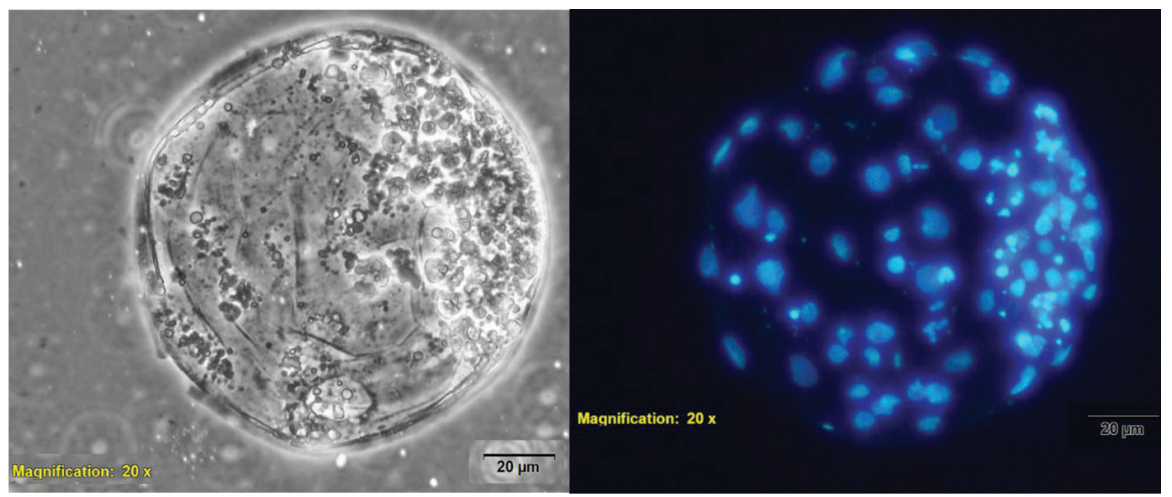

Figure 5. Pig blastocyst obtained in our laboratory because of in vitro fertilization by ICSI using freeze-dried sperm, and subjected to DAPI analysis - using light (left) and fluorescence microscopy (right) - the blue-stained nuclei of blastocyst

For horses, the ejaculated semen is mainly intended for lyophilization (Choi et al., 2011; Olaciregui et al., 2016; Oldenhof et al., 2017; Restrepo et al., 2019). After initial dilution in commercial diluents, the semen undergoes a detailed quality assessment or is directly diluted in lyophilization medium. The most commonly used media are based on TRIS-HCl + EDTA (Olaciregui et al., 2016; Oldenhof et al., 2017) or additionally enriched with the saccharides: glucose, sucrose, trehalose and bovine serum albumin (BSA) (Oldenhof et al., 2017), and TRIS-HCl + EGTA (Olaciregui et al., 2016) or DMEM/F12 (Choi et al., 2011; Restrepo et al., 2019). These prepared stallion sperm samples are subjected to one-stage (Choi et al., 2011; Restrepo et al., 2019) or two-stage freeze-drying (Oldenhof et al., 2017). The lyophilization time for stallion semen is from 10 to 48 hours (Choi et al., 2011; Oldenhof et al., 2017; Restrepo et al., 2019), at a constant pressure of 0.13 to $0.15 \mathrm{mBar}$ (Choi et al., 2011; Restrepo et al., 2019), or a variable pressure from 0.08 to $0.01 \mathrm{mBar}$ (Oldenhof et al., 2017) and a temperature range from $-75^{\circ} \mathrm{C}$ to $+20^{\circ} \mathrm{C}$ (Choi et al., 2011; Oldenhof et al., 2017; Restrepo et al., 2019). Lyophilisates are mainly stored in glass vials (Choi et al., 2011; Oldenhof et al., 2017; Restrepo et al., 2019) or cryotubes (Olaciregui et al., 2016), in various temperature ranges: from $4^{\circ} \mathrm{C}$ to $37^{\circ} \mathrm{C}$ (Choi et al., 2011; Olaciregui et al., 2016; Oldenhof et al., 2017), including RT (Restrepo et al., 2019). The storage time of lyophilisates is from 1 month to 1 year (Olaciregui et al., 2016; Oldenhof et al., 2017; Restrepo et al., 2019), or lyophilized semen is directly intended for fertilization (Choi et al., 2011). After rehydration, the freeze-dried stallion semen 
undergoes basic quality assessment, including computer-assisted microscopic assessment by the SCA system (Restrepo et al., 2019) or SpermVision (Oldenhof et al., 2017) and several analyses regarding the degree of sperm chromatin damage (e.g., chromatin dispersion test using the comet assay) (Olaciregui et al., 2016; Oldenhof et al., 2017) and chromatin fragmentation test using acridine orange staining (Choi et al., 2011; Olaciregui et al., 2016; Oldenhof et al., 2017). Moreover, the following parameters are also examined: the potential of the sperm mitochondrial membrane (Restrepo et al., 2019), degree of violation of the sperm cytoplasmic membrane using yellow luciferase staining (Oldenhof et al., 2017), propidium iodide (Oldenhof et al., 2017; Restrepo et al., 2019), lipid peroxidation in spermatozoa (Restrepo et al., 2019), trehalose concentration in sperm cells (Oldenhof et al., 2017) and mechanical damage to spermatozoa due to ice crystal formation and fluid solidification (evaluation by transmission microscopy) (Oldenhof et al., 2017).

The effectiveness of the stallion freeze-drying process is mainly evaluated based on the DNA fragmentation index (DFI). Restrepo et al. (2019) observed a very low percentage $(0.02)$ of spermatozoa showing nuclear chromatin fragmentation compared with vitrified (0.12) and frozen semen (0.03). A slightly higher DFI of stallion semen was noted by Choi et al. (2011) by performing three cycles of lyophilization and showing a slight increase in sperm with damaged chromatin (mean value: 5.6). In turn, research by Oldenhof et al. (2017) showed high DFI in freeze-dried stallion sperm stored for 2 weeks and 1 month (68\% and 95\%, respectively).

Horses are the only species of farm animals in which two live offspring were obtained after embryo transfer from oocytes fertilized by the ICSI method using freeze-dried sperm (Choi et al., 2011). The authors used sperm extract, introducing it in a small amount simultaneously with a single sperm into the oocyte cytoplasm. The phospholipase C zeta (PLC zeta) contained in the sperm extract is involved in the oocyte activation during fertilization and accelerates the whole process. Additionally, Choi et al. (2011) showed that stallion nuclear chromatin in spermatozoa is characterised by exceptional stability in relation to the lyophilization process, and additional activation of oocytes after injection is required for further embryonic development after ICSI.

\section{Pet animals}

Among the animals accompanying humans, sperm lyophilization studies were performed in dogs and cats. Dog semen for lyophilization is obtained from the spermrich fraction of the ejaculate (Watanabe et al., 2009; Olaciregui et al., 2015), while cat semen is obtained from the epididymis after castration (Ringleb et al., 2011). Similar to farm and laboratory animals, semen undergoes initial microscopic assessment or is intended directly for dilution in lyophilization medium based mainly on TRIS-HCl solution with the addition of EGTA (Watanabe et al., 2009; Ringleb et al., 2011; Olaciregui et al., 2015) or EDTA (Olaciregui et al., 2015). Semen samples are lyophilized in one (Ringleb et al., 2011) or two stages (Watanabe et al., 2009; Olaciregui et al., 2015) in the temperature range from $-68^{\circ} \mathrm{C}$ to $+20^{\circ} \mathrm{C}$ (Ringleb et al., 2011; Olaciregui et al., 2015) or at a constant temperature of $-30^{\circ} \mathrm{C}$ (Watanabe et al., 2009) and constant pressure of $0.016 \mathrm{mBar}$ (Ringleb et al., 2011) or a variable 
range from 0.053 to $0.001 \mathrm{mBar}$ (Watanabe et al., 2009; Olaciregui et al., 2015). The example lyophilization time for cat sperm is 4 hours (Ringleb et al., 2011). The lyophilisates obtained are stored in cryotubes (Ringleb et al., 2011; Olaciregui et al., 2015) or glass ampoules (Watanabe et al., 2009) at $-80^{\circ} \mathrm{C}$ (Watanabe et al., 2009; Ringleb et al., 2011), and $4^{\circ} \mathrm{C}$ or at RT (Olaciregui et al., 2015) for 1 month, 5 months or up to one year (Watanabe et al., 2009; Ringleb et al., 2011; Olaciregui et al., 2015). After reconstruction, the cat or dog sperm were evaluated for the degree of chromatin dispersion (Olaciregui et al., 2015) or morphological assessment using SEM (Ringleb et al., 2011).

The ICSI efficiency in pet animals was investigated for a cat, in which cleavage was achieved at approximately $28 \%$. Compared with the results of in vitro fertilization by ICSI with fresh semen, the ICSI efficiency with freeze-dried semen was significantly lower, and embryo development stopped at the 8-cell stage. The authors rightly commented on the need to improve lyophilization methods for cat semen and in vitro culture of oocytes and embryos (Table 1).

\section{Wild animals}

As with dogs and cats, in wild animals, sperm lyophilization is not a common method for the long-term preservation of male gametes. Until now, semen from the following species has been lyophilized: chimpanzee and giraffe (ejaculated semen) (Kaneko et al., 2014) and jaguar, weasel, long-haired rat (Kaneko et al., 2014) and marsupials (epididymal spermatozoa) (Czarny et al., 2009). Semen samples subjected to microscopic evaluation are diluted in a lyophilization medium based on TRIS solution with the addition of EDTA (Kaneko et al., 2014), for marsupials a trehalose solution or modified TRIS solution is prepared (Czarny et al., 2009). Semen is lyophilized in one step at a pressure of 0.2-0.3 mBar for 4 hours (Czarny et al., 2009; Kaneko et al., 2014). The lyophilisates obtained are stored in glass ampoules (Czarny et al., 2009; Kaneko et al., 2014), at $4^{\circ} \mathrm{C}$ or RT for several months (Czarny et al., 2009; Kaneko et al., 2014). To assess the sperm quality after lyophilization, a live/ dead viability test with SYBR-14/propidium iodide staining was used, and sperm morphology was assessed using Bryan staining, or an assessment of the level of apoptosis in spermatozoa was performed using the TUNEL method (Czarny et al., 2009).

\section{Conclusions}

Freeze-drying as an alternative method of the long-term conservation of mammalian semen has been used in biobanking and can help maintain the biodiversity of many species of farm and wild animals. Additionally, postmortem, ejaculated or epididymal sperm and fresh or previously frozen semen can be used for lyophilization. Because an important factor affecting IVF effectiveness is proper sperm motility, semen with reduced motility or nonmotile can be intended for freeze-drying and then for fertilization using the ICSI method. In this set of experiments, we managed to obtain embryos that reached the blastocyst stage and achieved full foetal development after ET.

The in vitro fertilization efficiency of ICSI is influenced by many factors associated with the lyophilization process, explaining why more research is required to 
develop the optimal lyophilization and ICSI procedure for a given animal species. A still unsolved problem associated with freeze-drying is the loss of sperm motility, which precludes the use of freeze-dried semen in IVF as well as in field conditions for female insemination. Additionally, during and after lyophilization, and then during storage of lyophilisates, sperm chromatin is often damaged in spermatozoa because of the action of pressure, temperature, free radicals, active endonucleases and other unknown chemical and physical factors (Restrepo et al., 2019; Gajda et al., 2019).

The fragmentation of sperm chromatin results in a reduced ability of freeze-dried spermatozoa to fertilize oocytes, which are associated with other abnormalities such as the impaired release of SOAF responsible for oocyte activation and biochemical changes in the cytoplasmic membrane of spermatozoa that compromise penetration into the cytoplasm of the sperm MPGF, initiates cell nucleus transformation sperm in functional male pronuclei (Kwon et al., 2004).

Therefore, many research teams have focused on identifying chemicals that can protect sperm DNA from harmful factors and positively affect the results of fertilization by the ICSI method using freeze-dried sperm. The group of proposed chemical compounds included chelating substances such as EDTA, EGTA, antioxidants, (e.g. $\mathrm{RA}$ ) or some metal ions (e.g. $\mathrm{K}^{+}$or $\mathrm{Na}^{+}$). Many experimental results have indicated a beneficial effect of the abovementioned substances and a decrease in the percentage of spermatozoa, as demonstrated by the quality assessment of the fragmentation of nuclear chromatin.

In contrast to frozen semen, which requires a constant, low storage temperature to ensure relative stabilisation of the genetic material, lyophilisates can be stored at various temperatures. This possibility prompted scientists to investigate the tolerance of the DNA of freeze-dried sperm to different temperatures. Wakayama et al. (2019) observed that single or multiple freezing at $-30^{\circ} \mathrm{C}$ or in liquid nitrogen and then heating the sperm of lyophilized mice to RT does not cause morphological changes in sperm and allows for obtaining live offspring as a result of in vitro fertilization using ICSI and ET. Similar results were achieved by single or multiple incubation at $95^{\circ} \mathrm{C}$ and even $100^{\circ} \mathrm{C}$ and $120^{\circ} \mathrm{C}$ lyophilized mouse sperm. Such a high temperature in a short time of exposure does not cause serious damage to the sperm nucleus (Wakayama et al., 2019).

In turn, Kamada et al. (2018) investigated the effect of RT and vacuum storage of lyophilisates on the DNA tolerance of freeze-dried sperm. The obtained results indicate that freeze-dried sperm stored in RT and under vacuum are characterised by a lower percentage of sperm chromatin damage and an increased ability to fertilize mouse oocytes (Kamada et al., 2018).

Based on the obtained results, a high or low ambient temperature and RT do not adversely affect sperm DNA and allowed live offspring to be obtained that reached adulthood. Furthermore, the use of, for example, vacuum-sealed ampoules in which lyophilisates are stored, additionally improved the efficiency of in vitro fertilization using the ICSI method and the number of live birth pups (Kamada et al., 2018; Wakayama et al., 2019).

Despite many different biotechnological procedures to ensure the stability of genetic material derived from freeze-dried spermatozoa, the in vitro fertilization ef- 
ficiency of ICSI using freeze-dried spermatozoa in some animal species remains unsatisfactory and requires further research and attempts to find optimal solutions. However, obtaining live offspring in this way suggests - after refining the freezedrying and the ICSI method more widely, using lyophilization as a method of the long-term preservation of male genetic material in the future.

\section{Acknowledgement}

We extend special thanks to Eng. Lechosław Gajda for valuable comments on the description of technical aspects of the freeze-drying process.

\section{References}

A bd a 11 a H., H i r a b a y a s h i M., H o ch i S. (2009). The ability of freeze-dried bull spermatozoa to induce calcium oscillations and resumption of meiosis. Theriogenology, 71: 543-552.

Anzalone D.A., Palazzesea L., I usoa D., Martinob G., Loi a P. (2018). Freeze-dried spermatozoa: An alternative biobanking option for endangered species. Anim. Reprod. Sci., 190: $85-93$.

A rav A., Id d a A., N i e d d u S.M., N a t a n Y., L e d d a S. (2018). High post-thaw survival of ram sperm after partial freeze-drying. J. Assist. Reprod. Genet., 35: 1149-1155.

Benedict M. (1905). The determination of water in foods and physiological preparations. Arn. J. Physiol., 13: 309-329.

Bhowmick S., Zhu L., McGinnis L., Lawitts J., Nath B.D., Toner M., Biggers J. (2003). Desiccation tolerance of spermatozoa dried at ambient temperature: Production of fetal mice. Biol. Reprod., 68: 1179-1786.

B i a ly G., S m i th V. R. (1957). Freeze-drying of bovine spermatozoa. J. Dairy Sci., 40: 739-745.

Choi Y.H., Varner D.D., Love C.C., Hartman D.L., H in richs K. (2011). Production of live foals via intracytoplasmic injection of lyophilized sperm and sperm extract in the horse. Reproduction, 142: 529-538.

Czarny N.A., Harris M.S., De Iuli is G.N., Rodger J.C. (2009). Acrosomal integrity, viability, and DNA damage of sperm from dasyurid marsupials after freezing or freeze drying. Theriogenology, 72: 817-825.

Domingo P., Olacireguia M., Gonzáleza N., De Blasb I., Gila L. (2018). Long-term preservation of freeze-dried rabbit sperm by adding rosmarinic acid and different chelating agents. Cryobiology, 81: 174-177.

Dos Santos Mora is M.L.G., de Brito D.C.C., Pinto Y., Silva L.M., Vizcarra D.M., S i lva R.F., Cibin F.W.S., Cam pello C.C., A lves B. G., A raú jo V.R., P in to F.Ch., Pes soa O.D.L., Figueiredo J.R., Rodrigues A.P.R. (2019). Natural antioxidants in the vitrification solution improve the ovine ovarian tissue preservation. Reprod. Biol., 19: 270-278.

Elliott G.D., Lee P-Ch., Paramore E., van Vorst M., Comizzoli P. (2015). Resilience of oocyte germinal vesicles to microwave-assisted drying in the domestic cat model. Biopreserv. Biobank, 13: 164-171.

Flosdorf E.W., Mudd S. (1935). Procedure and apparatus for preservation in "lyophile" form of serum and other biological substances. J. Immunol., 29: 389-425.

G a j d a B., R a j s k a I. (2014). Current and potential use of cryopreservation of farm animal embryos and oocytes (in Polish). Rocz. Nauk. PTZ, 10: 1-23.

Gajda B., Katska-Ksiazkiewicz L., Ryńska B., Bochenek M., S morag Z. (2007). Survival of bovine fibroblasts and cumulus cells after vitrification. Cryo Letters, 28: 271-279.

Gajd a B., R om e k M., Grad I., Krzyszt of ow ic z E., B ry la M., S morag Z. (2011). Lipid content and cryotolerance of porcine embryos cultured with phenazine ethosulfate. Cryo Letters, 32: 349-357.

Gajda B., Skrzypczak-Zielińska M., Gawrońska B., Słomski R., Smorąg Z. 
(2015). Successful production of piglets derived from mature oocytes vitrified using OPS method. Cryo Letters, 36: 8-18.

Gajda L., Cegła M., Rajska I., Gajda B. (2019). Effect of media on the DNA integrity of freeze-dried boar spermatozoa: preliminary study. Proc. 5th Winter Workshop of The Society for Biology of Reproduction, Zakopane, Poland, 13-15.02.2019, p.110.

Garcia A., Gil L., Malo C., Martine z F., Kershaw - Young C., d e B las I. (2014). Effect of different disaccharides on the integrity and fertilising ability of freeze-dried boar spermatozoa: a preliminary study. Cryoletters, 35: 277-285.

H a $\mathrm{m}$ m e r B.W. (1911). A note on the vacuum desiccation of bacteria. J. Med. Res., 24: 527-530.

Hara H., Abdalla H., Morita H, Kuwayama M., Hirabayashi M., Hochi S. (2011). Procedure for bovine ICSI, not sperm freeze-drying, impairs the function of the microtubule-organizing center. J. Reprod. Dev., 57: 428-432.

Hara H., Tagiri M., Hwang I.-S., Takahashi M., Hirabayashi M., Hochi S. (2014). Adverse effect of cake collapse on the functional integrity of freeze-dried bull spermatozoa. Cryobiology, 68: 354-360.

Hirabayashi M., Kato M., Ito J., Hochi S. (2005). Viable offspring derived from oocytes intracytoplasmically injected with freeze-dried sperm heads. Zygote, 13: 79-85.

H i r a b a y a s h M., K a to M., I to J., H o ch i S. (2007). Viable rat offspring derived from oocytes intracytoplasmically injected with freeze-dried sperm heads. Zygote, 13: 79-85.

Hochi S., Watanabe K., Kato M., Hirabayashi M. (2008). Live rats resulting from injection of oocytes with spermatozoa freeze-dried and stored for one year. Mol. Reprod. Dev., 75: $890-894$.

Holt W.V. (2000). Fundamental aspects of sperm cryobiology: the importance of species and individual differences. Theriogenology, 53: 47-58.

H ong x i a L., H a i x i a C., X ing p ing G., H u a i x i u W. (2018). Cryovial monolayer vitrification for ovarian tissue cryopreservation. Cell Tissue Bank, 19: 149-154.

I to D., Wakayama S., Ka mada Y., Shibasaki I., Kamimura S., O oga M., Wakaya$\mathrm{ma}$ T. (2019). Effect of trehalose on the preservation of freeze-dried mice spermatozoa at room temperature. J. Reprod. Dev., 65: 353-359.

K a mada Y., Wa ka y a ma S., Shiba sa ki I., I to D., Kamimura S., O oga M., Wakaya $\mathrm{m}$ a T. (2018). Assessing the tolerance to room temperature and viability of freeze-dried mice spermatozoa over long term storage at room temperature under vacuum. Sci. Rep., 8: 10602.

K a n e k o T. (2015). Simple sperm preservation by freeze-drying for conserving animal strains. Method. Mol. Cell. Biol., 1239: 317-329.

K a n e k o T., N a k a g a t a N. (2005). Relation between storage temperature and fertilizing ability of freeze-dried mouse spermatozoa. Comparative Med., 55: 140-144.

K a n e k o T., S e r i k a w a T. (2012). Successful long-term preservation of rat sperm by freeze-drying. PLoS One, 7: 35043.

Kan e k o T., Whitt ing h m D.G., Yan a g i m a ch i R. (2003). Effect of pH value of freeze-drying solution on the chromosome integrity and developmental ability of mouse spermatozoa. Biol. Reprod., 68: 136-139.

Ka n e k o T., K i m ura S., N a ka gat a N. (2007). Offspring derived from oocytes injected with rat sperm, frozen or freeze-dried without cryoprotection. Theriogenology, 68: 1017-1021.

K a n e k o T., K i m u r a S., N a k a g a t a N. (2009). Importance of primary culture conditions for the development of rat ICSI embryos and long-term preservation of freeze-dried sperm. Cryobiology, 58: 293-297.

K a n e k o T., I t o H., S a k a mo to H., O n u m a M., I n o u e- M u r a y a m a M. (2014). Sperm preservation by freeze-drying for the conservation of wild animals. PLoS One, 9: e113381.

K a s a i M., M u k a i a T. (2004). Cryopreservation of animal and human embryos. Reprod. Biomed. Online, 9: 164-170.

Kawa se Y., Suzuki H. (2011). Study on freeze-drying as a method of preserving mouse sperm. J. Reprod. Dev., 57: 176-182.

K a w a s e Y., A ray a H., K a ma da N., J is hage K. (2005). Possibility of long-term preservation of freeze-dried mouse spermatozoa. Biol. Reprod., 72: 568-573.

Kaw a se Y., Hani Y., Kamada N., Jishage K., Suzuki H. (2007 a). Effect of pressure at 
primary drying of freeze-drying mouse sperm reproduction ability and preservation potential. Reproduction, 133: 841-846.

Kawase Y., Tachibe T., Jishage K-I., Suzuki H. (2007 b). Transportation of freeze-dried mouse spermatozoa under different preservation conditions. J. Reprod. Dev., 53: 1169-1174.

K a w a s e Y., Wad a N.A., J is hag e K. (2009). Evaluation of DNA fragmentation of freeze-dried mouse sperm using a modified sperm chromatin structure assay. Theriogenology, 72: 1047-1053.

Keskintepe L., Pacholczyk G., Machnicka A., Norris K., Akif Curuk M., $\mathrm{Kh}$ an I., B rackett B.G. (2002). Bovine blastocyst development from oocytes injected with freeze-dried spermatozoa. Biol. Reprod., 67: 409-415.

$\mathrm{Kus}$ a kabe H. (2019). Production of mouse fetuses using spermatozoa exposed temporarily to high temperature or continuously to room temperature after freeze-drying in $\mathrm{Na}^{+}$-free $/ \mathrm{K}^{+}$-rich EGTA buffer. Cryobiology, 87: 105-109.

$\mathrm{K}$ u s a k a b e H., Ta t e n o H. (2011). Characterization of chromosomal damage accumulated in freezedried mouse spermatozoa preserved under ambient and heat stress conditions. Mutagenesis, 26: $447-453$.

Kusakabe H., Tateno H. (2017). Prevention of high-temperature-induced chromosome damage in mouse spermatozoa freeze-dried using $\mathrm{Ca}^{2+}$ chelator-containing buffer alkalinized with $\mathrm{NaOH}$ or KOH. Cryobiology, 79: 71-77.

Kusakabe H., Szczygiel M.A., Whittingham D.G., Yanagimachi R. (2001). Maintenance of genetic integrity in frozen and freeze-dried mouse spermatozoa. Proc. Natl. Acad. Sci. U.S.A., 98: 13501-13506.

K w o n I.K., P a r k K.E., N i w a K. (2004). Activation, pronuclear formation, and development in vitro of pig oocytes following intracytoplasmic injection of freeze-dried spermatozoa. Biol. Reprod., 71: $1430-1436$.

Lee P-Ch., Ada m s D.M., A melk in a O., White K.K., A moretti L.A., Whitaker M.G., Comizzoli P. (2019). Influence of microwave-assisted dehydration on morphological integrity and viability of cat ovarian tissues: First steps toward long-term preservation of complex biomaterials at supra-zero temperatures. PLoS One, 14: 1-23.

Li X.X., Diao Y.F., We i H.J., Wang S.Y., Cao X.Y., Zhang Y.F., Chang T., Li D.L., K i m M.K., X u B. (2017). Tauroursodeoxycholic acid enhances the development of porcine embryos derived from in vitro-matured oocytes and evaporatively dried spermatozoa. Sci. Rep., 7: 6773.

Liu J.L., Kusakabe H., Chang Ch.Ch., Suzuki H., Schmidt D.W., Julian M., Pfeffer R., Bormann Ch.L., Cindy Tian X., Yanagimachi R., Yang X. (2004). Freeze-dried sperm fertilization leads to full-term development in rabbits. Biol. Reprod., 70: $1776-1781$.

L i u J., L e e G.Y., L a w itts J.A., To ne r M., B ig g er s J.D. (2014). Live pups from evaporatively dried mouse sperm stored at ambient temperature for up to 2 years. PLoS One, 9: 1-7.

Loi P., Matsukawa K., Ptak G., Clinton M., Fulka J., Nathan Y., Arav A. (2008). Freeze-dried somatic cells direct embryonic development after nuclear transfer. PLoS One, 8: $1-6$.

Martins C.F., Báo S.N., D ode M.N., Correa G.A., Rumpf R. (2007 a). Effects of freezedrying on cytology, ultrastructure, DNA fragmentation, and fertilizing ability of bovine sperm. Theriogenology, 67: 1307-1315.

Martin s C.F., D ode M.N., B á o S.N., Ru mp f R. (2007 b). The use of the acridine orange test and the TUNEL assay to assess the integrity of freeze-dried bovine spermatozoa DNA. Genet. Mol. Res., 6: 94-104.

Men N.T., Kikuchi K., Nakai M., Fukuda A., Tanihara F., Noguchi J., Kaneko H., Linh N.V., N g u y en B.X., N a g a i T., Ta ji m a A. (2013). Effect of trehalose on DNA integrity of freeze-dried boar sperm, fertilization, and embryo development after intracytoplasmic sperm injection. Theriogenology, 80: 1033-1044.

Men N.T., Kikuchi K., Furusawa T., Dang-Nguyen T.Q., Nakai M., Fukuda A., J un ko N., K a n e k o H., Vi e t L.N., N g u y en B.X., Ta j i ma A. (2016). Expression of DNA repair genes in porcine oocytes before and after fertilization by ICSI using freeze-dried sperm. Anim. Sci. J., 87: 1325-1333. 
Mercati F., Domingo P., P a squariello R., Dall' Aglio C., Di Michele A., Forti K., Coc c i P., B o it i C., Gil L., Zeran i M., M a r a nes i M. (2019). Effect of chelating and antioxidant agents on morphology and DNA methylation in freeze-drying rabbit (Oryctolagus uniculus) spermatozoa. Reprod. Domest. Anim., 55: 29-37.

Mu n e to T., H o r i u c h i T. (2011). Full-term development of hamster embryos produced by injecting freeze-dried spermatozoa into oocytes. J. Mamm. Ova. Res., 28: 32-39.

Nakagawa Y., Kan e k o T. (2019). Rapid and efficient production of genome-edited animals by electroporation into oocytes injected with frozen or freeze-dried sperm. Cryobiology, 90: 71-74.

Nakai M., Kashiwazaki N., Takizawa A., Maedomari N., Ozawa M., Noguchi J., Kane k o H., Shino M., Ki k u chi K. (2007). Effects of chelating agents during freeze-drying of boar spermatozoa on DNA fragmentation and on developmental ability in vitro and in vivo after intracytoplasmic sperm head injection. Zygote, 15: 15-24.

Narbutt O., Dąbrowski H.P., Dąbrowska G. (2017). The process of freeze-drying, its wide applications and defense mechanisms against dehydratation (in Polish). Ed. Biol. Środ., 2: 20-29.

O l a c ir e gu i M., G il L., L u ñ o V., J e r e z R.A., G o n z a l e z N. (2014). Use of rosmarinic acid as antioxidant on boar sperm freeze-drying. Reprod. Domest. Anim., 49: 105.

Ol a c ir egui M., L uño V., G onzal e z N., D e B la s I., Gil L. (2015). Freeze-dried dog sperm: Dynamics of DNA integrity. Cryobiology, 71: 286-290.

Olaciregui M., Luño V., Martí J.I., Aramayona J., Gil L. (2016). Freeze-dried stallion spermatozoa: evaluation of two chelating agents and comparative analysis of three sperm DNA damage assays. Andrologia, 48: 900-906.

O la c ir e gu i M., L uño V., D o ming o P., G on zále z N., Gi 1 L. (2017 a). In vitro developmental ability of ovine oocytes following intracytoplasmic injection with freeze-dried spermatozoa. Sci. Rep., 24: 1096.

Olaciregui M., Luño V., Domingo P., González N., de Blas I., Gil L. (2017 b). Chelating agents in combination with rosmarinic acid for boar sperm freeze-drying. Reprod. Biol., 17: $193-198$.

Old e nh of H., Zhang M., N arten K., B igalk J., S y d y k o v B., Wolkers W.F., S i e m e H. (2017). Freezing-induced uptake of disaccharides for preservation of chromatin in freeze-dried stallion sperm during accelerated aging. Biol. Reprod., 97: 892-901.

Pa lazzes e L., Gos álvez J., Anzal on e D.A., L o i P., S a r a g us ty J. (2018). DNA fragmentation in epididymal freeze-dried ram spermatozoa impairs embryo development. J. Reprod. Dev., 64: $393-400$.

Patrizio P., L o i L., A rav A. (2012). Lyophilization and rehydration of bovine oocytes after vitrification: a new technological breakthrough. Fertil. Steril., 23: 46.

P o 1 g e C., S m it h A.U., P a r ke s A.S. (1949). Revival of spermatozoa after vitrification and dehydration at low temperature. Nature, 164: 666-667.

$\mathrm{R}$ a j s k a I. (2015). Intra-cytoplasmic sperm injection (ICSI) as an alternative to standard in vitro fertilization in pigs (in Polish). Rocz. Nauk. PTZ, 11: 55-66.

R a m i r e z J.S., C a ñ i zare s J. (2003). Deshidratacion de la papa mediante liofilización atmosferica. Ecuador: Escuela de Ingenierı Quimica, Universidad Central Del Ecuador, Escuela De Ingeniería Química, Quito-Ecuador.

Re stre p o G., Var e la E., D u qu e J.E., Gó m e z J.E., R oj a s M. (2019). Freezing, vitrification, and freeze-drying of equine spermatozoa: impact on mitochondrial membrane potential, lipid peroxidation and DNA integrity. J. Equine Vet. Sci., 72: 8-15.

R ingleb J., Wa u ri ch R., Wib b e lt G., S tre i ch W.J., J e w gen ow K. (2011). Prolonged storage of epididymal spermatozoa does not affect their capacity to fertilise in vitro-matured domestic cat (Felis catus) oocytes when using ICSI. Reprod. Fert. Develop., 23: 818-825.

S a ragusty J., Arav A. (2011). Current progress in oocyte and embryo cryopreservation by slow freezing and vitrification. Reproduction, 141: 1-19.

S h a c k e 11 L.F. (1909). An improved method of desiccation, with some applications to biological problems. Arn. J. Physiol., 20: 325-340.

Sherman J.K. (1954). Freezing and freeze-drying of human spermatozoa. Fertil. Steril., 5: $357-371$. 
Silva H.V.R., d a Silva A.M., Le e P-Ch., B rito B.F., S ilva A.R., d a Silva L.D., Comizz o li P. (2020). Influence of microwave-assisted drying on structural integrity and viability of testicular tissues from adult and prepubertal domestic cats. Biopreserv. Biobank, 18: 415-424.

Waka y a m T., Yanagimachi R. (1998). Development of normal mice from oocytes injected with freeze-dried spermatozoa. Nat. Biotechnol., 16: 639-641.

Wa ka y m a S., Kam a da Y., Yamanaka K., Kohda T., Suzuki H., Shimazu T., Motoki N.T., Ikuko O., N ag a matsu A., K a mimura S., Nagat omo H., Mizutani E., I s h in o F., Y a n o S., Wa k a y m a T. (2017). Healthy offspring from freeze-dried mouse spermatozoa held on the International Space Station for 9 months. Proc. Natl. Acad. Sci. U.S.A., 114: $5988-5993$.

Waka y m a S., I to D., Kamad a Y., Yonemura S., O og a M., Kishigami S., Wakaya$\mathrm{ma}$ T. (2019). Tolerance of the freeze-dried mouse sperm nucleus to temperatures ranging from $-196^{\circ} \mathrm{C}$ to $150^{\circ} \mathrm{C}$. Sci. Rep., 9: $1-9$.

Ward M.A., Kaneko T., Kusakabe H., Biggers J.D., Whittingham D.G., Yanagi$\mathrm{m}$ a ch i R. (2003). Long-term preservation of mouse spermatozoa after freeze-drying and freezing without cryoprotection. Biol. Reprod., 69: 2100-2108.

W at an ab e H., A s a no T., A b e Y., F u k u i Y., S u zuk i H. (2009). Pronuclear formation of freezedried canine spermatozoa microinjected into mouse oocytes. J. Assist. Reprod. Genet., 26: 531-536.

Received: 13 VII 2020

Accepted: 11 XII 2020 